\section{INMIGRANTES EN CAMPAMENTOS EN CHILE: ¿MECANISMO DE INTEGRACIÓN $O$ EFECTO DE EXCLUSIÓN?'1}

Ernesto López-Morales ${ }^{2}$, Pablo Flores Pineda ${ }^{3}$ y Hernán Orozco Ramos ${ }^{4}$

\section{Resumen}

En la actualidad, Chile enfrenta nuevamente el incremento del número de hogares viviendo en campamentos (asentamientos informales autoconstruidos) en las periferias de las principales ciudades del país, esta vez no solo con una componente de hogares previamente allegados, sino también con hogares con aumentados costos en gastos de vivienda y una alta tasa de población migrante latinoamericana. Este artículo explora posibles factores causales, tales como una economía de suelo y vivienda socioeconómicamente excluyente, en la que prevalece el acceso por la vía del arriendo como única opción formal, pero que se hace insustentable para

\section{IMMIGRANTS IN SETTLEMENTS IN CHILE: A MECHANISM OF INTEGRATION OR THE EFFECT OF EXCLUSION?}

\author{
Ernesto López-Morales², Pablo Flores Pineda ${ }^{3}$ and \\ Hernán Orozco Ramos ${ }^{4}$
}

\section{Abstract}

Chile is once again facing an increased number of households living in informal settlements in the peripheries of main cities. However, this time the phenomenon does not only refer to non-paying lodgers, but also households who have experienced increased housing costs and high rates of Latin American immigrant population. This paper explores possible causal factors such as a socioeconomically exclusionary land and housing market that leaves substandard renting as the only 'formal' option available for the poor but one that is unsustainable for the most vulnerable population. Urban informality is also seen as a grey area 
los más vulnerables, así como el desarrollo de la informalidad urbana como una zona gris donde se confrontan políticas públicas, derechos denegados y geografías de oportunidades; ello, en contraste con la idea de los campamentos como vías para la obtención de beneficios estatales. El artículo presenta y analiza datos desagregados de la encuesta CIS TECHO-Chile a campamentos realizada en 2017, de alcance nacional, y se observa en mayor detalle la situación de campamentos en la ciudad de Antofagasta, en el norte de Chile, ciudad de tasas elevadas de "campamentación".

PALABRAS CLAVE: CAMPAMENTOS; INMIGRANTES; MERCADO DE VIVIENDA; EXCLUSIÓN; VIVIENDA SOCIAL.

Recibido: 14-03-2018

Aceptado: 27-08-2018

1 Centro de Investigación Social (CIS) TECHO-Chile; Facultad de Arquitectura y Urbanismo, Universidad de Chile; Proyecto Fondecyt Regular 1151287; Proyecto COES (Conicyt Fondap 15130009).

2 Chile. Universidad de Chile, Facultad de Arquitectura y Urbanismo Departamento de Urbanismo. Centro de Estudios del Conflicto y la Cohesión Social (COES). ORCID: http://orcid.org/00000003-2878-7143. Correo electrónico: elopez@uchilefau.cl.

3 Chile. Centro de Investigaciones Sociales, TECHO-Chile. ORCID: http://orcid.org/0000-0001-5251-1636. Correo electrónico: pablo.flores@techo.org.

4 Chile. Departamento de Planificación y Ordenamiento Territorial, Universidad Tecnológica Metropolitana. ORCID: http://orcid. org/0000-0002-4326-9099. Correo electrónico: h.orozcor@utem.cl. where public policies, the denial of social rights and geographies of opportunities are confronted in contrast to previous ideas of informal settlements as a way to obtain state benefits in Chile. This paper presents and analyses disaggregated data from the 2017 CIS TECHO-Chile national survey of informal settlements and delivers a qualitative analysis on settlements in the city of Antofagasta, north Chile, a city with a growing number of informal settlements.

\section{KEYWORDS: INFORMAL SETTLEMENTS; IMMIGRANTS; HOUSING MARKET; EXCLUSION; SOCIAL HOUSING.}

Received: 14-03-2018

Accepted: 27-08-2018

1 Center for Social Research (CIS) TECHO-Chile; Faculty of Architecture and Urban Planning, University of Chile; Regular Fondecyt Project No 1151287; COES Project (Conicyt Fondap project No 15130009).-

2 Chile. University of Chile, Faculty of Architecture and Urban Planning, Department of Urban Planning. Center for Social Conflict and Cohesion Studies (COES). ORCID: http://orcid. org/0000-0003-2878-7143. Email: elopez@uchilefau.cl.

3 Chile. Center for Social Research, TECHO-Chile. ORCID: http:// orcid.org/0000-0001-5251-1636. Email: pablo.flores@techo.org.

4 Chile. Department of Territorial and Land Use Planning, Metropolitan University of Technology. ORCID: http://orcid.org/00000002-4326-9099. Email: h.orozcor@utem.cl. 


\section{Introducción}

Campamento es la denominación en Chile para los asentamientos informales. El Ministerio de Vivienda y Urbanismo (2011) define campamento como un asentamiento de al menos ocho familias, con un indicador de contigüidad habitacional (superficie del terreno del asentamiento medida en $\mathrm{m}^{2}$ ) menor o igual al número de viviendas multiplicados por 350. La definición implica también una tenencia irregular del terreno (arriendos sin contratos, cedidos y que sean propiedad privada, municipal o estatal ocupada de hecho) y acceso irregular a uno o más de servicios de energía eléctrica, agua y/o solución sanitaria. Este tipo de asentamiento se caracteriza por una posesión del terreno y acceso a servicios básicos que no se realizan a través de mecanismos convencionales, sino que la comunidad del campamento se organiza para satisfacer sus necesidades, lo que en muchos casos origina un sentido de pertenencia al territorio y una identidad colectiva ${ }^{5}$.

La política de vivienda chilena de las décadas de 1990 y 2000 se tradujo en una producción masiva de viviendas sociales, localizadas y estandarizadas según las posibilidades de obtención de ganancias del sector (privado) de la construcción y gestión inmobiliaria, con un rol de subsidio por parte del Estado. Esa política logró reducir el déficit de un millón de viviendas acumulado durante la dictadura que finalizó en 1990 (Besoain y Cornejo, 2015). Sin embargo, hoy en Chile, y en el entendido de que vivienda adecuada es más que una unidad de vivienda social de estándar mínimo subsidiada (Rodríguez y Sugranyes, 2005), el número de hogares en déficit de vivienda adecuada ha vuelto a empinarse a más de 500.000 viviendas (Cámara Chilena de la Construcción, 2017), con una población residiendo en campamentos que también ha aumentado. En 2011, eran casi 27.400 hogares en esta condición, pero en 2017 eran ya 40.540 (CIS TECHO-Chile, 2017). Esto es, un 8\% del déficit nacional.

En los últimos años, la variable de hogares inmigrantes carenciados ha sido incidente en el resurgimiento de campamentos, con una tasa de hogares inmigrantes que llega actualmente al $28 \%$ de la población viviendo en campamentos a nivel nacional. En los dos últimos periodos intercensales (19922017) Chile ha multiplicado por 10 la presencia de hogares inmigrantes, con una tasa de feminización de la jefatura de hogar creciente (actualmente en $55 \%$ del total) y población de migración reciente,

5 Según muestra la Encuesta CIS TECHO-Chile (2017), el 50\% de los hogares de campamentos se organiza en Comités de Vivienda. 
juvenil y sin hijos. Es posible establecer entonces la siguiente pregunta relativa al resurgimiento de los campamentos en Chile: ise enfrenta el país a una falla de mercado (temporal) de vivienda producto del diseño incorrecto de política pública de acceso a la vivienda, o se trata de causas más profundas con nuevas implicancias por conocer y estudiar?

Las condiciones de vida en estos asentamientos son difíciles. Estudios de CIS TECHO-Chile (2015) indican que, a nivel nacional, un $76 \%$ de hogares que vive en condición de campamento no tiene acceso formal al agua potable, un 91\% no tiene alcantarillado y un 48\% no tiene acceso formal a la electricidad. La tasa de educación escolar incompleta supera el 65\% para los adultos, y la de analfabetismo es de un $12,5 \%$, muy por encima de la media nacional. Además, son frecuentes en los campamentos los hogares con ingreso infra-remunerados y empleo informal o extremadamente flexibilizado, donde un $58,7 \%$ de quienes trabajan lo hacen en empleos informales, ganando un $60 \%$ menos del sueldo mínimo (CIS TECHO-Chile, 2015).

Radicarse en un campamento hoy aparece para una parte de la sociedad como una solución factible a un mercado "formal" privado de arriendo de vivienda que para muchos es inaccesible económicamente. Son frecuentes los casos de abuso y maltrato por parte de propietarios o administradores de conventillos y cités, y las situaciones de hacinamiento son especialmente altas para lo que refiere a la vivienda de hogares inmigrantes en el mercado "formal" (las comillas en el uso del término son, por ende, deliberadas). Los adjetivos no sobran para denunciar los mal llamados "guetos verticales" y la proliferación de conventillos con altos precios de arriendo por pieza en las ciudades del país (Sabatini, 2017); asimismo, según CASEN 2015, los hogares viviendo en modalidad de allegados son un $240 \%$ más que en 2000 (183.000 familias) a nivel país (Ministerio de Desarrollo Social [MIDESOC], 2015).

Ambas situaciones -vivienda antigua y vivienda nueva precarias, en modalidad de allegamiento o hacinamiento, como únicas opciones de localización "formal" para hogares vulnerables- provienen de las fallas de regulación estatal sobre los mercados privados de vivienda y sobre todo los precios de oferta en Chile (López Morales, 2016). Sin embargo, los migrantes internacionales además viven en un limbo institucional, bajo una categoría de ciudadanía precarizada, en último lugar de la lista de espera para la consagración de su derecho a la vivienda (Stefoni, Leiva y Bonhomme, 2017). Sumado a lo anterior, la estigmatización y violencia etnorracial existente, especialmente en la zona norte del país, que es la que ha atraído mayor número de inmigrantes pobres, lleva aparejado el estigma adicional de la localización "informal" y una percepción subjetiva notablemente superior de menor cohesión y mayor conflicto barrial y estigma territorial (Méndez, Otero, López, Link y Castillo, 2017). 
El objetivo de este artículo es, en una primera parte cuantitativa, indagar en las relaciones existentes entre funcionamiento del mercado de la vivienda e incremento del número de migrantes residiendo en campamentos a nivel país. En una segunda parte cualitativa, el artículo se enfoca en el caso de la zona norte del país y la ciudad de Antofagasta en particular, analizando las causas y experiencias del campamento desde la perspectiva de los residentes migrantes.

\section{Metodología}

La metodología utilizada en este estudio comprende dos partes, a saber, la utilización de herramientas cuantitativas (encuesta de nivel nacional) y cualitativas (entrevistas enfocadas en una región). Para la parte cuantitativa se utilizan los resultados de una encuesta realizada a hogares que viven en campamentos de aquellas regiones del país donde ha aumentado la cifra de campamentos entre los años 2011 y 2016, en un total de 60 campamentos ubicados en siete regiones de Chile: Tarapacá, Antofagasta, Atacama, Valparaíso, Metropolitana, Biobío y Los Lagos (Figura 1). La encuesta fue realizada a 1.785 hogares, alcanzando un porcentaje de cobertura que permite lograr un margen de error del 5\% para muestras regionales. El cuestionario estructurado, que tuvo un total de 45 preguntas, fue confeccionado por profesionales de
CIS TECHO-Chile y piloteado y ajustado por los autores de este artículo, previo a su aplicación definitiva en los campamentos. El cuestionario fue aplicado a jefes de hogar o cónyuges que fueron anteriormente avisados del día y hora de la encuesta por los dirigentes vecinales en contacto con CIS TECHO-Chile, institución que además facilitó encuestadores voluntarios previamente capacitados.

Las principales variables a medir por la encuesta fueron los indicadores sociodemográficos de los hogares, las causas previas de la llegada al campamento, los motivos de habitar en un campamento y la trayectoria migratoria. Entre las preguntas más relevantes se encuentran las condiciones habitacionales de la vivienda anterior al campamento, el suceso que lleva al desplazamiento urbano al campamento, entre otras de tipo "espacial". Los datos fueron analizados a través de procesos estadísticos simples como frecuencias, porcentajes y tablas de contingencias (apoyados por el programa estadístico SPSS v.21).

Para la parte cualitativa se utilizan los resultados de un estudio encomendado por el Ministerio de Desarrollo Social (MIDESOC) a TECHO-Chile, con el fin de conocer la trayectoria de migrantes que llegan a vivir en campamentos de la Región de Antofagasta. Se aplicó un total de 41 entrevistas semiestructuradas y grupos focales-, en tres campamentos con presencia de familias migrantes ( $\mathrm{Ta}$ bla 1). Dentro de cada campamento se escogieron 
TABLA 1. POBLACIÓN ENTREVISTADA EN CAMPAMENTOS DE ANTOFAGASTA.

\begin{tabular}{|c|c|c|c|c|c|c|}
\hline Campamento & Año de fundación & Instrumento & Hombres & Mujeres & Edad (prom.) & Participantes \\
\hline \multirow{2}{*}{ Mujeres Unidas } & \multirow{2}{*}{2007} & Entrevista & 0 & 4 & 35 & \multirow{2}{*}{11} \\
\hline & & Grupo focal & 3 & 4 & 34 & \\
\hline \multirow{4}{*}{ Chile Nuevo } & \multirow{4}{*}{1991} & Entrevista & 0 & 3 & 38 & \multirow{2}{*}{10} \\
\hline & & Grupo focal & 3 & 4 & 36 & \\
\hline & & Entrevista & 0 & 3 & 35 & \multirow{2}{*}{10} \\
\hline & & Grupo focal & 2 & 5 & 34 & \\
\hline \multirow{2}{*}{ Juanita-Cruchaga } & \multirow{2}{*}{1975} & Entrevista & 2 & 3 & 32 & \multirow{2}{*}{10} \\
\hline & & Grupo focal & 3 & 2 & 29 & \\
\hline TOTAL & & & 13 & 28 & & 41 \\
\hline
\end{tabular}

Fuente: Elaboración propia.

a los entrevistados por medio de la metodología "bola de nieve", aunque los dirigentes también recomendaron quiénes serían idóneos tanto para entrevistas como para grupos focales. Debido a los objetivos de la investigación, solo se trabajó con entrevistados extranjeros, realizando un muestreo teórico (muestra no probabilística) con saturación de la información.

Los campamentos seleccionados fueron aquellos presentes en un catastro actualizado de campamentos de la comuna de Antofagasta y que presentaran las siguientes características: presencia de familias migrantes de distintos países, familias migrantes de un solo país y familias chilenas, y campamentos de familias migrantes sin chilenos.
El análisis de los datos fue a través de "teoría fundamentada", método de análisis de datos cualitativos que busca generar conceptos e hipótesis como resultados de la aplicación del instrumento (Hernández, Fernández y Baptista, 2010), es decir, se busca generar una teoría de alcance medio con relación a la conceptualización del fenómeno de migrantes en campamentos en la zona norte del país. Por medio de un proceso inductivo y de comparación constante, se generó una teoría explicativa del fenómeno social. El procesamiento se realizó por medio del software ATLAS.ti, facilitando la codificación. Las citas a discursos seleccionadas se referencian con pseudónimos. 


\section{Encuadre teórico}

A partir de la dictadura militar (1973-1990), bajo la lógica neoliberal de extrema individualización, focalización social y Estado subsidiario, los pobladores anteriormente vistos como sujetos de cultura rural precaria (Vekemans y Fuenzalida, 1976) o como agentes con potencial revolucionario (Castells, 1974) pasaron a ser vistos agentes individuales receptores de subsidios para la adquisición de viviendas en el mercado privado. Los pobladores se debatieron entre el preservar una capacidad de autoorganización y desarrollo de vínculos fuertes al interior de las comunidades ya establecidas, y la pérdida de cohesión social en los nuevos entornos residenciales, a menudo segregados, carenciados y vulnerables, que fueron promovidos por la dictadura y los posteriores gobiernos democráticos de la Concertación (suelo barato y localización menos ventajosa como principales factores considerados por los desarrolladores). Así, el estándar residencial deficiente logrado quedó en espera de la consolidación de entornos con servicios y bienes públicos básicos, promesa aún incumplida para cientos de miles de hogares pese a los esfuerzos de paliar este déficit cualitativo desde mediados de la década de 2000 en adelante (Rodríguez y Sugranyes, 2005).

En la década de 2000 se generó un nuevo incremento del número de hogares en campamentos, cuya causa principal fue la decisión individual racional de los hogares (no necesariamente "pobres", aunque sí despolitizados) que establecían una suerte de "chantaje" al Estado subsidiario democrático (Brain, Prieto y Sabatini, 2010), con el fin de acelerar la obtención de subsidios a la vivienda en propiedad, acceder a una buena localización no periférica y mejorar en el intertanto su geografía de oportunidades al estar ubicados más cerca de sus redes. En rigor, "una estrategia residencial [individual] para ejercer un mayor control espacial de su situación residencial" (Brain, Prieto y Sabatini, 2010, p. 139).

Según Brain, Prieto y Sabatini (2010), el Estado respondió con urgencia a esta presión, ya que la asociación campamento-pobreza está implícita aunque nunca cuestionada por evidencia- en sus políticas públicas. La tesis de estos autores logró dar una explicación para el año 2010, cuando Chile aún no enfrentaba un déficit de vivienda tan serio como el que emergería ocho años más tarde, y cuando la variable de inmigración internacional masiva aún no hacía su estreno en el país. Hoy, se sabe que la situación es distinta y que se requieren explicaciones de mayor complejidad ante la creciente "campamentación"; requiriéndose también una mirada, por una parte, menos institucional, y, por la otra, más enfocada en las realidades microlocales de estos asentamientos, asumiendo también el carácter internacional de sus agentes como una variable importante para su análisis. 
Por su parte, trabajos provenientes de la psicología ambiental y la geografía cultural han intentado explicar el fenómeno de hogares que retornan al campamento, postulando la existencia de una resistencia de los sujetos a los procesos de subjetivación e individualización generados por el tránsito hacia la vivienda social formal provista por el Estado, resistencia que implica un volcamiento hacia un pasado idealizado (vida en el campamento) donde los vínculos sociales, dejados atrás, se perciben como más solidarios y menos anómicos (Morales et al., 2017; Besoain y Cornejo, 2015). La noción de retorno al campamento conllevaría una utopía de un lugar que no existe, pero que se añora. Si bien se trata de una hipótesis plausible, estos trabajos no cuantifican la incidencia de la variable "añoranza" en el fenómeno de crecimiento de campamentos, ni las condiciones materiales políticoeconómicas que lo explican en Chile.

Los campamentos de hoy están fuertemente marcados por el nivel de vulnerabilidad, concebida como procesos estructurales que configuran la amenaza de caer o mantenerse en situación de pobreza. En concordancia con las teorías de la vulnerabilidad (Kaztman, 1999; Busso, 2001; Moreno Crossley, 2008), el proceso que lleva a una persona o familia a habitar en campamentos tendría relación con los "procesos estructurales que configuran situaciones de fragilidad, precariedad, indefensión o incertidumbre" (González, 2009, p. 2), es decir, los individuos o familias que se encuentran en una situación de vulnerabilidad, por razones de un hecho detonante (pérdida del trabajo, ruptura familiar o problemas económicos) o también por la migración por razones de precariedad económica y búsqueda de mayor estabilidad a largo plazo, conllevaría en muchos casos habitar en campamentos. Este proceso es llamado "campamentación", puesto que da cuenta de que cualquier persona o familia que se encuentra en este estado, al tener un accidente que afecta sus posibilidades de cubrir sus necesidades de habitabilidad, puede entrar o ingresar en situación de campamento (CIS TECHO-Chile, 2015).

Por otra parte, respecto al fenómeno de la inmigración, tomar la decisión de abandonar el país de origen es resultado de un conjunto de factores sociales. Solimano (2008) ha mencionado cuatro principales factores para la realidad latinoamericana: 1) las brechas de ingreso y desarrollo entre los países, 2) las crisis de crecimiento y crisis financieras, 3) el desempleo, salarios e informalidad del mercado laboral, y 4) otros determinantes, tales como las crisis políticas, las redes de apoyo familiar y amistades, las políticas de inmigración de los países, y la distancia geográfica, entre otros. Existe consenso de que el proceso de "campamentación" refuerza formas de exclusión social. 
Las primeras acepciones de exclusión social, como complemento o incluso contrapeso a las visiones más deterministas de "pobreza", refirieron hace cincuenta años a la población desprotegida de los sistemas de protección social del contexto europeo, mutando luego en las décadas de 1980 y 1990 al concepto de "nueva pobreza" de gran influencia explicativa para una América Latina sumida en la "economía social de mercado" (Apaolaza, 2018). El concepto de exclusión se trata, de cualquier forma, de una factura de un segmento humano respecto al total de la sociedad. Etimológicamente, la exclusión se trata de una reclusión o encerramiento (claudere) afuera de (ex) un "todo" que implica la sociedad. Tal como lo manifiesta Joan Subirats (2004), la exclusión social opera en distintos niveles, que para la realidad específica de los campamentos se visibilizan respecto a su habitabilidad, y también se comprende en un sentido geográfico, como la denegación de posibilidades o capacidades materiales de las personas para ocupar espacios en la ciudad que, en condiciones normales, podrían ser ocupados por estas (Slater, 2009).

Algunas economías de suelo y vivienda operarían entonces como mecanismos centrales de exclusión social y geográfica (López Morales, 2015), una desigualdad en el acceso a determinados recursos, que es determinada por la localización y habitabilidad de los entornos residenciales (Apaolaza, 2018). Por su parte, Morales et al. (2017, p. 58) plantean que los campamentos no solo son formas de exclusión, sino también cumplirían una función de falsa inclusión en los márgenes físicos de la ciudad, al ser esenciales para la reproducción del sistema, "lugares heterotópicos [que] no producen nada por si mismos en tanto su densidad marginal es producto de [y funcional a] la ciudad formal".

Según registros del Centro de Investigación Social (CIS) de la Fundación TECHO-Chile, desde 2011 a 2016 la cifra de familias que llegan a vivir en este tipo de asentamientos ha aumentado en un 41,6\% (CIS TECHO-Chile, 2016). Este aumento también refleja que la realidad de los campamentos es dinámica. Al respecto, los principales incrementos del número de familias se concentran en las regiones de la macrozona norte de Chile y las ciudades metropolitanas del Gran Valparaíso y el Gran Concepción, siendo el número de hogares internacionales inmigrados que optan o son arrojados a esta situación también considerable. En este contexto, el presente estudio propone la hipótesis que el fenómeno de la migración extranjera se ve afectada por los procesos de la metamorfosis de la ciudad que se materializan en geografías de desigualdad, continuas explotaciones laborales, acrecentadas por otras explotaciones habitacionales. La información y análisis presentados a continuación validan esa hipótesis. 


\section{Campamentos y migrantes a nivel nacional. El complejo crecimiento de los campamentos en un contexto de alta migración}

En la última década, la política pública de vivienda ha evidenciado una disminución del déficit habitacional cuantitativo. Sin embargo, a pesar de estas cifras, el número de familias que viven en campamentos en Chile presenta una tendencia al alza desde el año 2011. Según la actualización del Catastro Nacional de Campamentos 2017 (CIS TECHO-Chile, 2017), existen 40.541 familias en 702 campamentos. Este aumento de un 48\% entre 2011 y 2017 tiene una expresión en la forma urbana en contextos regionales particulares, principalmente en la macrozona norte del país. Por ejemplo, en la Región de Antofagasta, el aumento es de un 487\%, en Tarapacá (extremo norte de Chile, donde se sitúa la ciudad de Iquique) la tasa es de un 104\%, y en el caso de Atacama, región extractiva minera cuya capital es Copiapó, la tasa es de un 131\% (Figura 1).

En un país donde un $88 \%$ vive en zonas urbanas (Instituto Nacional de Estadísticas [INE], 2017) y donde el déficit habitacional cuantitativo ha
FIGURA 1. CAMPAMENTOS ENCUESTADOS EN CHILE.

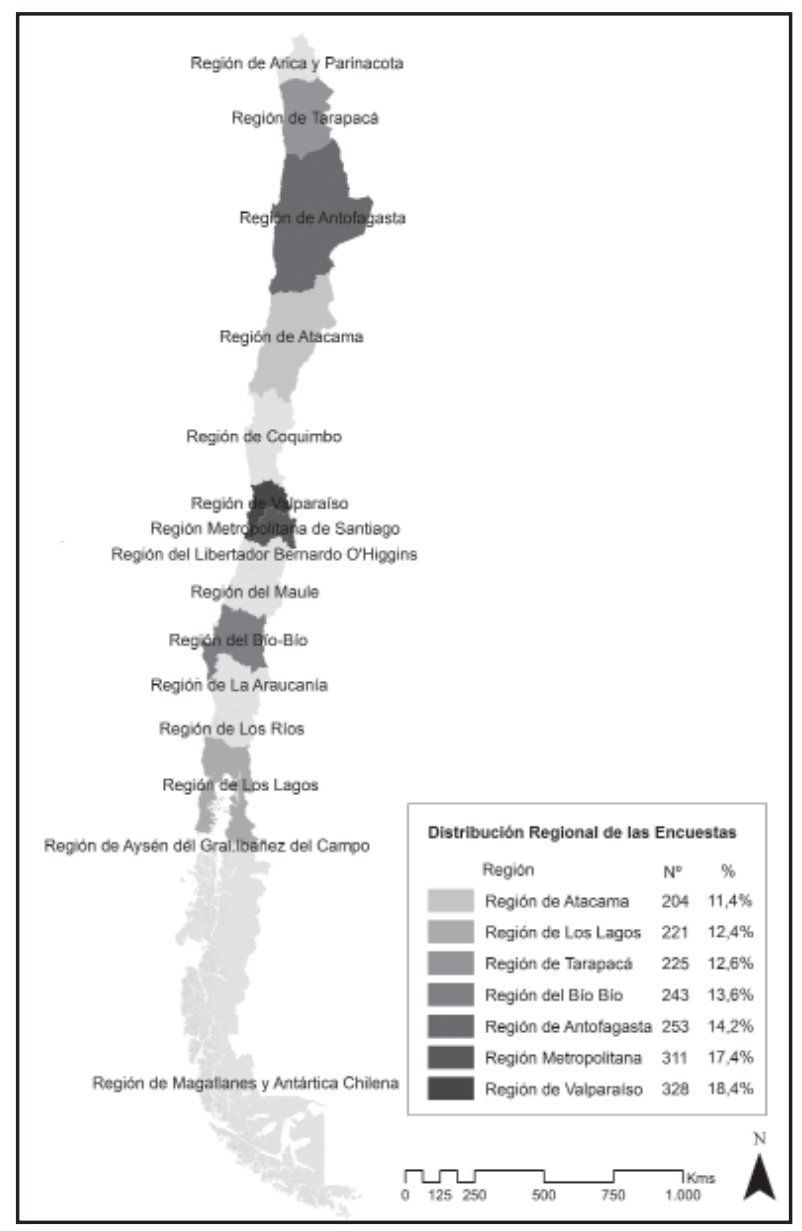

Fuente: Elaboración propia en base a Encuesta a Campamentos Migrantes CIS TECHO-Chile. 
experimentado una tendencia a la baja en los últimos casi 30 años, la pregunta acerca de las causas del aumento de los campamentos es compleja. Aproximarse a una respuesta implica reconocer una mirada local respecto al fenómeno, desde los procesos de producción social de nuestras ciudades, y también una mirada global, relacionada con migración económica. Un análisis de las cifras de campamentos permite identificar que entre 2016 y 2017 salieron de campamentos 2.600 familias, pero que al mismo tiempo llegaron a vivir en campamentos 4.600. De este total, 800 familias son extranjeras, formando parte de los 36 campamentos con población migrante registrados a nivel nacional (CIS TECHO-Chile, 2015). Los extranjeros en campamentos provienen principalmente de Bolivia (32,4\%), Colombia (32,3\%) y Perú $(27,1 \%)$. Parte importante de la población más reciente de inmigrantes, principalmente dominicanos, también accede a la vivienda por esta vía.

Las causas de la notoria llegada de familias extranjeras a campamentos son materia de inacabable indagación. En la última década, la migración extranjera ha experimentado un importante aumento en Chile. Según cifras oficiales, más de 746.000 extranjeros residen actualmente en el país, representando un $4,3 \%$ de la población (al año 2006, ese porcentaje era de solo un 1\%) (INE, 2017). Estudios indican que la gran mayoría de estos migrantes extranjeros opta por vivir en ciudades, como una estrategia para incorporarse rápidamente al mercado laboral y así poder enviar remesas a sus países de origen (Solimano, 2008). No obstante, si bien la mayoría de esta población opta por vivir en el Gran Santiago, hay una gran proporción que también habita en otras ciudades chilenas, específicamente en la zona norte del país, donde la intensiva explotación minera está estrechamente relacionada con mayores oportunidades laborales y también con un más desregulado crecimiento de las ciudades.

La encuesta ELSOC 2016 del Centro de Estudios del Conflicto y la Cohesión Social muestra desigualdades importantes en las regiones de Tarapacá, Antofagasta, Atacama y Coquimbo (Méndez et al., 2017) respecto al resto del país. Sin estar asociados a la variable "inmigración", los datos de la macrozona norte chilena muestran, en conjunto, un 34\% de sus habitantes que perciben sus barrios como territorialmente estigmatizados, en contraste con un 17\% de la zona centro-sur (incluye Santiago) y un 19\% de la zona sur. Respecto a las sensaciones de inseguridad barrial, el porcentaje para los habitantes de la zona norte es de un 30\%, casi el doble del centro-sur y sur. En niveles de confianza entre vecinos, apego al barrio, sociabilidad y apoyo social entre vecinos, las ciudades de la zona norte vuelven a mostrar resultados muy por debajo del promedio nacional.

Seguido de las comunas centrales del Gran Santiago, un grupo no menor de población extranjera vive en la ciudad de Antofagasta, alcanzando un 5,3\% de la población total de esa comuna. 
Caracterizada por su aporte a la economía nacional -a través de la extracción del cobre y su efecto en el desarrollo de un mercado inmobiliario acelerado por un exceso de capital disponible para inversión y una deficiente regulación- Antofagasta ha logrado posicionarse como la región con más alto PIB per cápita de Chile, alcanzando US\$37.205 (Banco Central de Chile, 2015). En ese marco, las características de la ciudad de Antofagasta se relacionan con los factores de atracción para la migración de tipo laboral, cuestión que permitiría acelerar la concreción de expectativas para el mejoramiento de la calidad de vida de extranjeros que optan por migrar de sus países de origen.

\section{Campamentos de migrantes en Chile: resultados de la Encuesta 2017}

La Encuesta a Campamentos Migrantes realizada por CIS TECHO-Chile muestra que, a nivel país, las nacionalidades de origen de las familias son las siguientes: un $72 \%$ son familias chilenas, mientras que un $10,8 \%, 7,51 \%$ y $6,78 \%$ corresponden a familias bolivianas, colombianas y peruanas, respectivamente. A nivel regional, en Antofagasta destacan el grupo de colombianos con un 44,01\% y los bolivianos con un 30\% del total de residentes en condición de campamentos. En Tarapacá (capital Iquique), las familias peruanas representan un 18\%, y en la Región Metropolitana de Santiago las familias dominicanas representan un 12,22\%.

A pesar de que la localización de la mayoría de estos campamentos data desde hace décadas, estos han tenido distintas olas de crecimiento y decrecimiento producto de las campañas de erradicación en base a subsidios de vivienda individuales y en menor medida colectivos, y por la llegada paulatina de nuevas familias por motivos de carácter económico, cultural, de acción colectiva, y fundamentalmente como una estrategia para solventar la falta de acceso a la vivienda (Rivas, 2013).

Es posible evidenciar dos periodos característicos de llegada a los campamentos encuestados en los últimos 20 años (Figura 2): el primero, entre 1998 a 2010, donde llegaron a campamentos el 14\% de las familias encuestadas, a razón de 20 familias por año, subiendo el promedio anterior del periodo 1978-1997, que era de tres familias por año. El segundo periodo característico va desde 2010 al presente (2016), donde arribaron a los campamentos estudiados 1.531 familias (169 familias por año). Del total de familias que arribaron a campamentos en este periodo, el 33,68\% tiene nacionalidad distinta a la chilena, con una tasa de arribo a razón de 57 familias al año. Además, destaca el año 2013 como el punto de inflexión de la curva de crecimiento de familias migrantes en proceso de campamentación. 


\section{FIGURA 2. NÚMERO DE FAMILIAS POR AÑO DE LLEGADA A CAMPAMENTOS.}

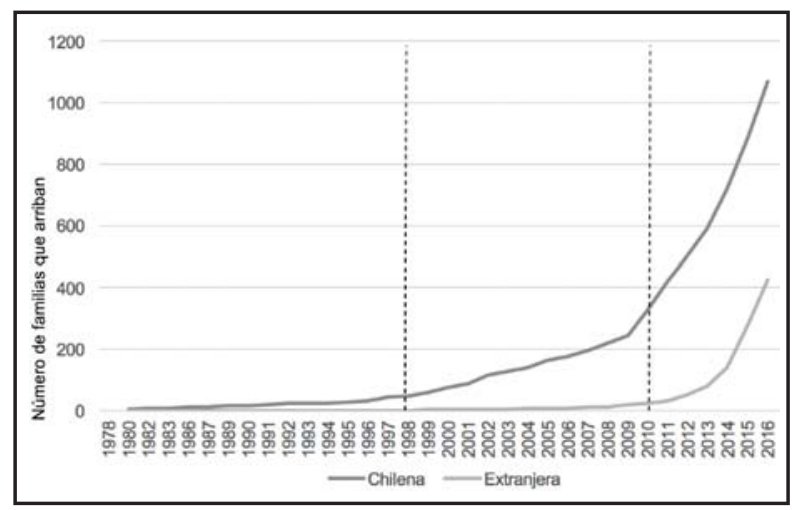

Fuente: Elaboración propia en base a Encuesta a Campamentos Migrantes CIS TECHO-Chile.

Otra de las condiciones relevantes de estos campamentos es el género de los jefes de hogar, considerando que un 57,37\% de los hogares tienen jefa (femenina) de hogar, y que incluso en comunidades extranjeras como la ecuatoriana y la boliviana estas cifras superan el 66\%. Este dato contrasta con Brain, Prieto y Sabatini (2010), quienes aducían para la década de 2000 una mayoría de jefaturas de hogar de sexo masculino, con ingresos por sobre la línea de la pobreza. Son estas condiciones también de especial interés para esta investigación, si se considera que raza, etnia y género son variables típicamente reconocidas como condiciones de vulnerabilidad e incluso marginación en los procesos de acceso a la vivienda (Farha, 2017).
El nivel educacional de los jefes de hogar del campamento bien podría ser otro factor que relativice las condiciones de vulnerabilidad observadas hace una década atrás. Un $71,26 \%$ de los jefes de hogar chilenos tiene educación media incompleta o inferior, mientras que solo un $56,76 \%$ de los jefes de hogar extranjeros está en esa condición (incluso un $4 \%$ de extranjeros en estos campamentos tiene estudios superiores; y en el caso de los dominicanos, un $13,2 \%$ tiene estudios terciarios).

La situación migratoria de los jefes de hogar también fue una variable observada. Antes de llegar al campamento, los hogares con residencia definitiva eran un $35,2 \%$, mientras que en la actualidad (ya en el campamento) el 68,54\% de los extranjeros, y especialmente el $77 \%$ de peruanos y bolivianos (como los grupos con mayor nivel de formalización) tienen residencia definitiva. Esto podría constatar, basados en las autodeclaraciones de la encuesta, que la regularización migratoria no es un factor relevante de vulnerabilidad y en rigor no tiene relación, o podría tener una relación inversa, con el proceso de campamentación que experimentan las familias. A ello se suma la situación laboral de estos grupos de carácter permanente $(51 \%)$, de forma remunerada (65\%) y contratada (71,82\%), valores sorprendentemente altos y que, en rigor, contradicen lo que intuitivamente se esperaba antes de hacer la encuesta. 
Sin embargo, en lo que respecta a la situación contractual en la tenencia de la vivienda, en el momento anterior a la llegada de los hogares al campamento, sí resulta posible evidenciar una condición de precariedad relacionada con la condición migratoria. Mientras que el $24,16 \%$ de los hogares chilenos arrendaba vivienda sin contrato, el $62,68 \%$ de los hogares extranjeros arrendaba bajo esa misma condición (tasa que para el caso de las familias bolivianas sube a 70,69\%).

Una característica que sí es común para todos los hogares encuestados es la precariedad habitacional que experimentaban antes de acceder al campamento. Los niveles generales de hacinamiento eran del 49\%, y de hacinamiento crítico del 20\%. Además, un 38,26\% de los hogares de estos campamentos, que en promedio se componen de 3,5 personas vivían en apenas una habitación, cuyo costo promedio superaba los $\$ 117.000$ (US\$ 180), lo que representa un $42 \%$ de un ingreso mínimo en Chile $^{6}$.

El porcentaje de los ingresos destinados al gasto familiar en vivienda (housing cost burden, en inglés), en el caso de estos hogares, específicamente gasto en arriendo, también marca una diferencia importante entre el antes y después de acceder al campamento. En promedio, los hogares que antes de acceder al campamento destinaban casi un $40 \%$ de sus ingresos al arriendo (en Chile, el

Sueldo mínimo en Chile al año 2018 es de $\$ 276.000$ (US\$ 425). máximo nivel de endeudamiento exigido por la banca para una hipoteca de vivienda es de un 25\% del ingreso familiar), disminuyeron ese porcentaje a un $18,73 \%$ una vez llegados al campamento (Tabla 2). Esta drástica reducción de costos asociados a la vivienda ocurre incluso en el contexto de una disminución de los ingresos promedio de los hogares, de $\$ 335.984$ (US $\$ 517$, antes del campamento) a $\$ 313.730$ (US\$ 483, después de llegar al campamento). Esta última reducción se explica probablemente por un acceso menos diversificado a empleos.

Indagada la razón principal que se tuvo para llegar al campamento (Figura 3a), las respuestas a nivel general de las familias muestran que en primer lugar se trata del alto costo del arriendo (46,72\%), siendo esta razón aún más marcada en el grupo extranjero $(74,12 \%)$ y especialmente para los colombianos (82,81\%). Muy por detrás se sitúan las pocas oportunidades laborales (14,96\%) y el endeudamiento (7,54\%). Para las familias chilenas, el segundo factor importante es la situación previa de allegamiento (29,18\%), siendo casi tan importante como el costo previo del arriendo (35,19\%).

Respecto de los "hechos que detonaron la decisión de mudarse a un campamento" (Figura 3b) el más recurrente tiene relación con el alza en el costo del arriendo (a nivel general, 27,04\%), donde 
TABLA 2. PORCENTAJE DE HOUSING COST BURDEN (HCB)*

\begin{tabular}{lcccccc}
\cline { 2 - 6 } Nacionalidad & \multicolumn{3}{c}{ Antes de llegar al campamento } & \multicolumn{3}{c}{ En el campamento } \\
\cline { 2 - 6 } & Total ingresos & $\begin{array}{c}\text { Costo de } \\
\text { arriendo }\end{array}$ & HCB & Total ingresos & $\begin{array}{l}\text { Costo de } \\
\text { arriendo }\end{array}$ & HCB \\
Chilena & $\$ 316.893$ & $\$ 120.609$ & $38.06 \%$ & $\$ 288.857$ & $\$ 71.060$ & $24.60 \%$ \\
Extranjera & $\$ 378.073$ & $\$ 160.180$ & $42.37 \%$ & $\$ 372.738$ & $\$ 48.853$ & $13.11 \%$ \\
Total & $\$ 335.984$ & $\$ 138.612$ & $41.26 \%$ & $\$ 313.730$ & $\$ 58.756$ & $18.73 \%$ \\
\hline
\end{tabular}

(*) Para traspasar a valores en US\$, dividir montos por 650 (tasa de cambio a junio de 2018).

Fuente: Elaboración propia en base Encuesta a Campamentos Migrantes CIS-Techo 2017.

nuevamente los grupos más afectados son los hogares extranjeros $(46,88 \%)$ y colombianos $(62,90 \%)$. Los siguen factores detonantes como problemas familiares (21,43\%), pérdida del trabajo (13,01\%), y desalojo de la vivienda previa (16,45\%).

Todo lo anterior se complementa con el reconocimiento de situaciones de abuso por parte de los arrendadores, experimentadas por los hogares migrantes previo a la llegada al campamento. Los cobros excesivos en los arriendos ocupan el primer lugar $(57,26 \%)$, seguido por condiciones injustas en los contratos $(28,22 \%)$, deterioro de la vivienda arrendada $(22,61 \%)$, y tratos injustos por ser extranjero (20,84\%). Ante este escenario, es posible plantear que la llegada a un campamento, más complejo que un mero reflejo de las condiciones de vulnerabilidad del migrante, o un mecanismo de presión al Estado, es una respuesta a las diversas formas de exclusión que presenta el mercado de la vivienda en Chile.

Sin embargo, la mayor parte de los encuestados $(69,41 \%)$ también considera que su "situación en general" ha mejorado respecto de su situación previa al campamento, mientras que solo un $16,75 \%$ cree que se ha mantenido, y apenas un $11,74 \%$ cree que ha empeorado. La tendencia es aún más marcada en el grupo de hogares extranjeros, que cree en un $73,91 \%$ de los casos que su situación, cualitativamente percibida, ha mejorado en un alto grado.

Es posible hacer referencia a algunos aspectos de la cotidianeidad que los encuestados reconocen también han mejorado, como por ejemplo el tamaño de la vivienda, la tranquilidad y seguridad del barrio, la capacidad de ahorro y la posibilidad de tener a los familiares y conocidos residiendo cerca, lo cual es especialmente importante para los 


\section{FIGURA 3. RAZONES Y HECHOS PARA MUDARSE AL CAMPAMENTO.}

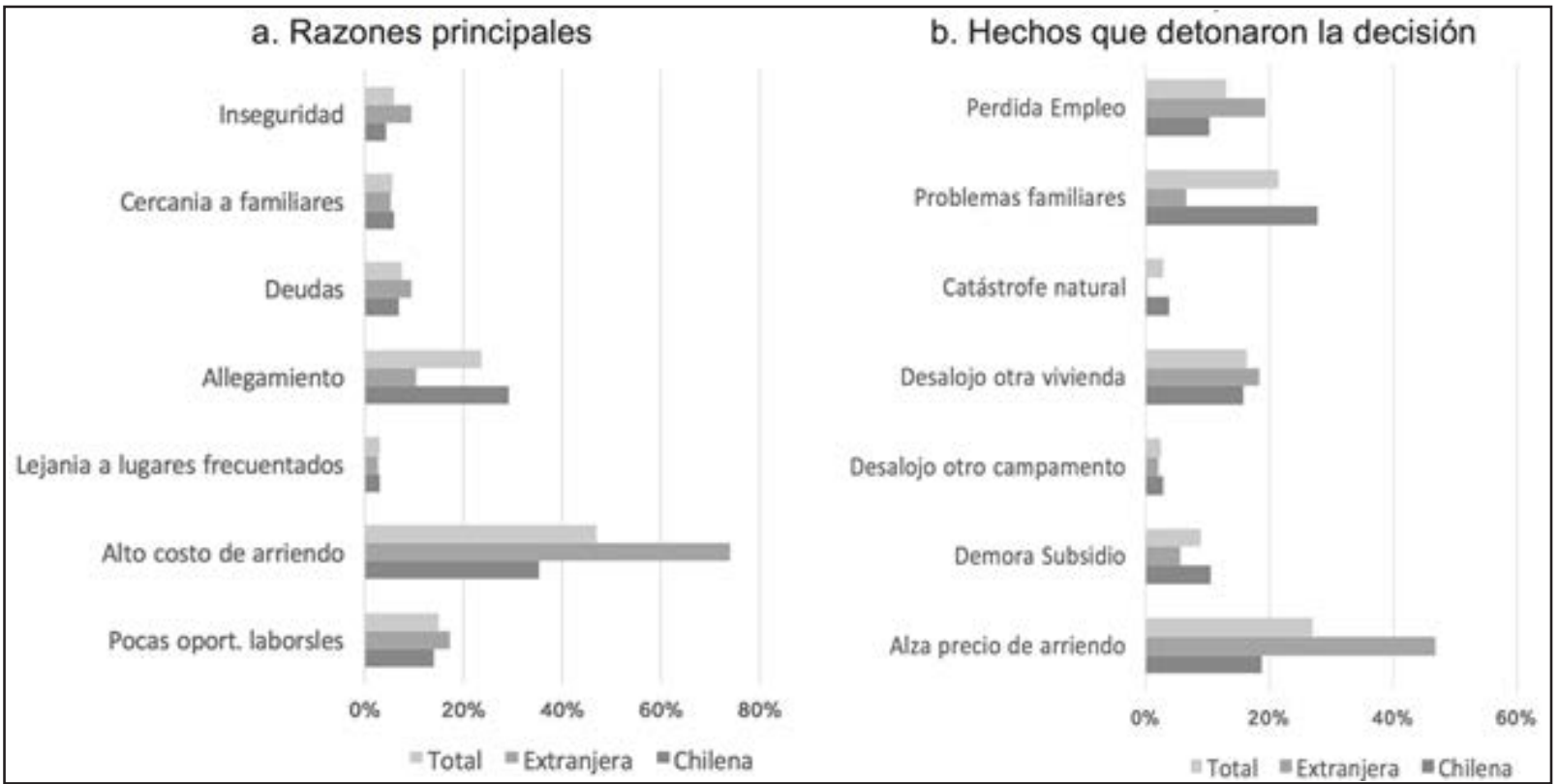

Fuente: Elaboración propia en base Encuesta a Campamentos Migrantes CIS TECHO-Chile 2017.

grupos extranjeros. Asimismo, en lo que respecta a aspectos que han empeorado, destacan el tener que enfrentarse con la contaminación ambiental, el alcoholismo, la drogadicción y la delincuencia (en el entorno urbano del campamento), además de la falta de servicios, lo cual es especialmente importante para la comunidad chilena. A pesar de que la llegada al campamento tiene costos asociados a una mayor exposición a problemas sociales y ambientales, esta pareciese ser una decisión considerablemente favorable en términos de la percepción del entorno habitacional (Morales et al., 2017).

Finalmente, en un contexto donde menos de un $3 \%$ de los migrantes encuestados desea retornar a su país de origen, es interesante conocer las expectativas habitacionales de los diferentes grupos ( $\mathrm{Fi}-$ gura 4). A un 65,27\% de los encuestados les gustaría tener una vivienda definitiva en la misma comuna, siendo apenas un 5,02\% quienes preferirían 
FIGURA 4. EXPECTATIVAS HABITACIONALES.

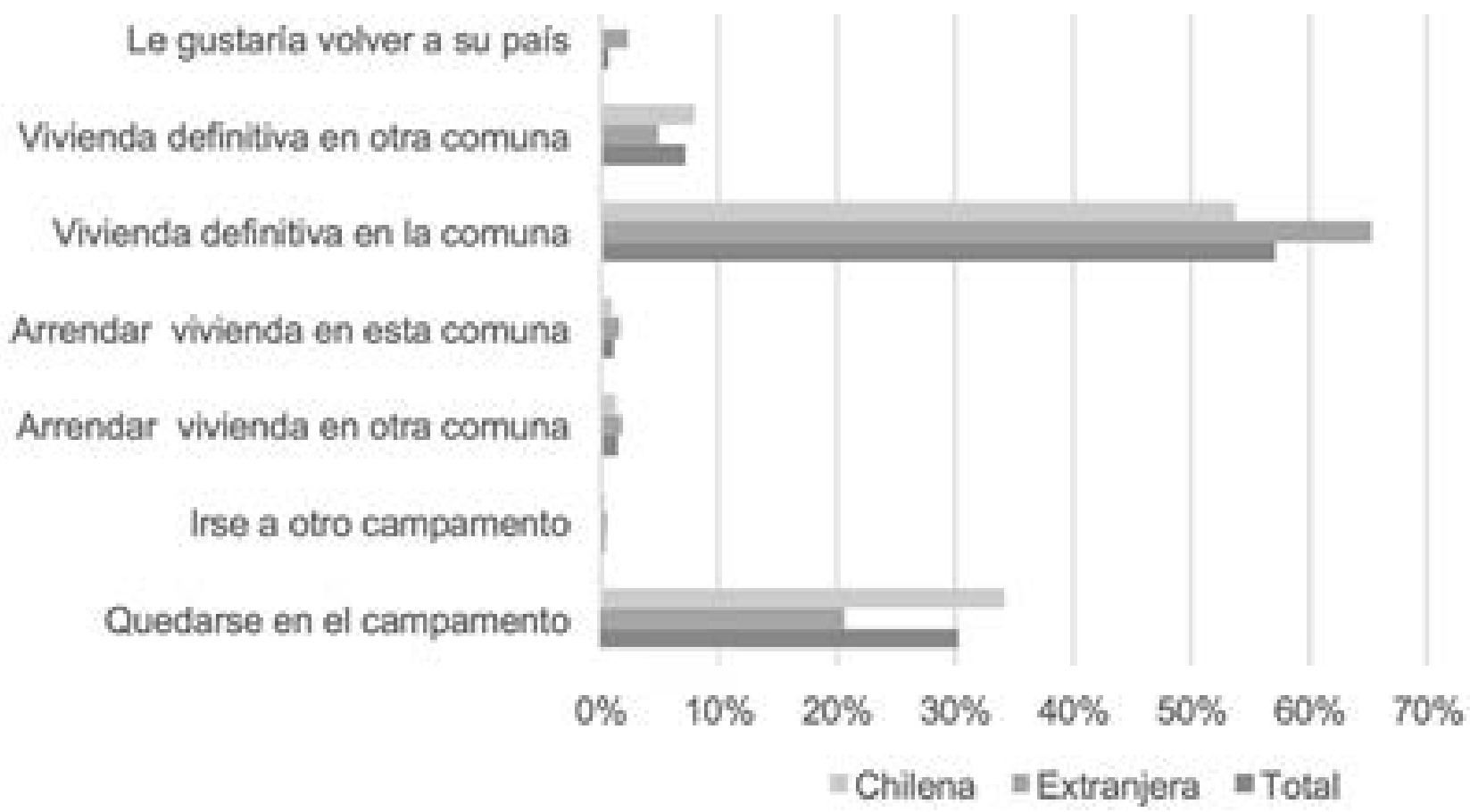

Fuente: Elaboración propia en base Encuesta a Campamentos Migrantes CIS TECHO-Chile (2017).

una vivienda definitiva en otra comuna. Entre las familias chilenas, esta última opción es algo más alta $(8.07 \%)$, aunque no tanto como la preferencia por quedarse en el campamento, que expresan un $34,26 \%$ de las familias nacionales. En paralelo destaca la bajísima preferencia por adoptar la condición de arrendatario, o volver a tenerla, ya sea en la misma comuna o en otra, lo que evidentemente tienen relación con el "anhelo" de la vivienda en propiedad, correspondiente con una aspiración de estabilidad financiera, y también con el temor de vivir nuevamente situaciones de vulnerabilidad, enfrentados a un mercado del arriendo altamente desregulado, y marginalizados de un mercado inmobiliario económicamente excluyente. 


\section{Aproximaciones al aumento de población migrante en campamentos de Antofagasta}

Las ciudades de la zona norte del país son las que presentan mayor crecimiento de campamentos, asociado a una también alta localización de familias migrantes. En tal sentido, se trata de un fenómeno a profundizar en la vinculación entre migración, vulnerabilidad y hábitat informal. Precisamente, el estudio cualitativo realizado en tres campamentos con población migrante en la ciudad de Antofagasta permite acercarse a posibles explicaciones causales referidas al incremento del fenómeno de la "campamentación" a nivel del país.

Antofagasta es la principal área urbana de la región homónima, albergando 361.000 habitantes (65.000 más que el año 2002) en poco más de 112.000 viviendas (INE, 2017). La economía regional se basa en la actividad minera, aunque también cada vez más fuertemente en los servicios y el mercado inmobiliario (Daher, 2003); lo anterior, junto a las condiciones de vulnerabilidad socionatural (ancho litoral acotado y zonas de riesgo por remoción en masa y aluviones) han sido las principales causas de la segregación urbana (Thodes Miranda, 2016). Esta ciudad, alargada de norte a sur, ha desarrollado un gradiente de estratificación socioeconómica oriente-poniente con predominancia de estratos
FIGURA 5. CAMPAMENTO EN ANTOFAGASTA.

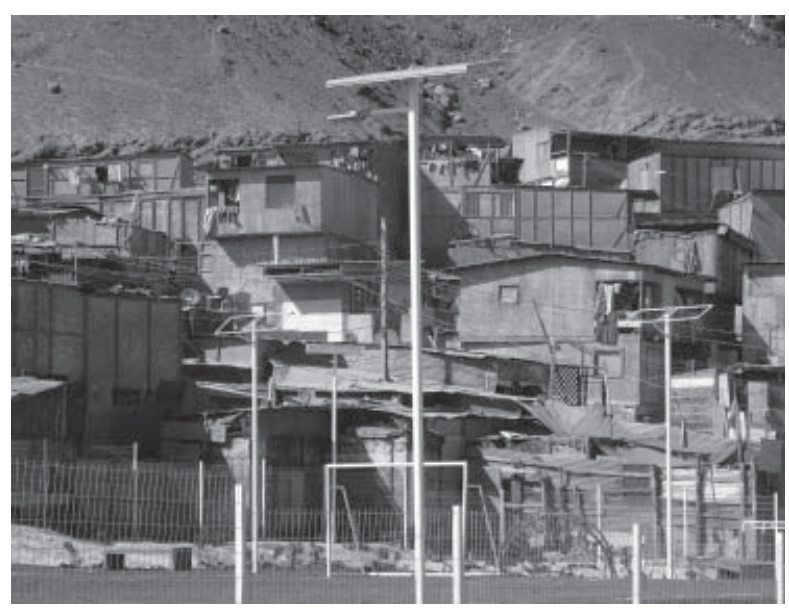

Fuente: autores.

medios y altos en el borde costero, de estratos medios en la planicie litoral, y de estratos bajos (entre ellos los campamentos) en contacto directo con el farellón costero y las condiciones de riesgo que este conlleva (Figura 5); además se observa una polarización de los estratos más bajo y más alto, con los primeros concentrándose al norte de la ciudad y los segundos concentrados en el extremo sur en condominios cerrados (Toro y Orozco, 2018).

Desde el año 2011 a la fecha, la cifra de familias en campamentos en la región de Antofagasta ha aumentado en un 487\%, llegando a las 6.229 familias en la actualidad (CIS TECHO-Chile, 2016). Según cifras del Gobierno Regional de Antofagasta, un 
59,8\% de la población que vive en campamentos declara ser extranjera (GORE Antofagasta, 2015).

$\mathrm{Al}$ analizar las entrevistas, los relatos de los migrantes no están alejados de los cuatro factores planteados por Solimano (2008), explicados anteriormente. La decisión de migrar tiene como antecedente la dificultad de las familias de concretar un mejoramiento en su calidad de vida, expresadas en la precarización laboral y que trae como resultado bajos salarios e informalidad en el mercado laboral en el país de origen. También se presentan situaciones del contexto político y social de los países, tales como la violencia e inseguridad a raíz de la delincuencia y la guerrilla.

"Vendíamos que el chancho, que el pollo y nos hemos acorralado de deudas. Y de la noche a la mañana dijimos: '¿Dónde nos vamos?'. No quiero estar aquí, que ya nos vienen a cobrar. Empezaron a llegar papeles de juicio y tomé la decisión de venirme yo sola para acá." (Lucía, 34 años, boliviana).

Otro aspecto identificado en las causas de salir del país también se relaciona con las experiencias de migración hacia Chile de amigos o conocidos. El campamento aparece como una oportunidad para concretar la reunificación familiar en un territorio que permite ciertas ventajas respecto a modalidades más "formales" de acceso a la vivienda. Por ejemplo, la posibilidad de poder juntarse con los suyos, y vivir en familia, modifica el perfil migratorio de los familiares que llegan después, no necesariamente con el fin de cumplir expectativas laborales, sino que de reunirse con quienes arribaron primero.

\section{LA BÚSQUEDA DEL TRABAJO Y EL TECHO}

Al momento de llegar al país, una de las principales necesidades se vincula a la búsqueda de un lugar donde residir y un trabajo con el cual conseguir ingresos. Es en ese momento donde aparece la dificultad de ingresar al mercado formal del trabajo, ya que al no tener redes de contactos o por problemas en la obtención de la regularización migratoria, se favorece la entrada al mercado informal del trabajo a partir de empleos subcalificados y donde existe explotación e incuso maltrato por causas raciales.

\footnotetext{
"En ocasiones había veces que uno sentía como que nos decían que habíamos dos morenitos, dos negritos, y siempre veíamos que las represalias eran más contra nosotros dos. Yo era maestra de cocina y se supone que a mí me contratan para ser maestra, y me exigían lavar platos, hacer el aseo o ponerme a servir en las mesas, aunque hubieran garzones." (Jennis, 43 años, colombiana).
}

Sin embargo, el mercado informal, aunque precario, permite obtener ingresos a corto plazo. Es con el paso del tiempo, la articulación de redes de conocidos o familiares, que al migrante le facilita el ingreso a empleos formales, con contrato de trabajo y seguridad social. Al considerar que las migraciones estudiadas tienen un carácter principalmente 
laboral, es que la esfera del trabajo determina en gran medida los procesos de inserción en el país.

En cuanto al acceso a la vivienda, existen modalidades de arriendo informal, sin contrato, que permite al arrendatario llevar el control de la relación y modificar los acuerdos a su interés. Muchas veces eso posibilita el mejoramiento de las viviendas, pero otras veces aparece el abuso y cobro excesivo por la vivienda. Esto se expresa también en los usos residenciales de los espacios habitacionales de los cités y viviendas tugurizadas. No tener la formalidad del arriendo lleva al cobro excesivo de necesidades básicas como el uso de agua caliente para la ducha, el cobro por persona que conforma el hogar, como también la restricción de los espacios, tales como el uso de los pasillos del sitio para la recreación de niños o adultos, la utilización de la electricidad en la noche, la entrada y la salida de la vivienda, entre otros. Esto también opera en las alzas injustificadas a los costos del alquiler. El hostigamiento del arrendatario por el pago del arriendo mensual y la amenaza ante el posible término del acuerdo, son prácticas identificadas por las experiencias de los entrevistados.

"(...) arriendan compartido, los arriendos aquí se volvieron a los famosos compartidos, por decir algo, vivo yo, viene una amiga, yo arriendo la misma pieza con mi amiga, y nos toca dormir hasta en la misma cama (...) Eso lo han creado las mismas personas, de la demanda de tantos extranjeros aquí, entonces, claro, ellos ven la oportunidad de hacer su plata también, porque, con tanto extranjero, dicen, cobremos por cada persona que llegue a la pieza (...)" (Carmen, 28 años, colombiana).

Para el acceso a la vivienda existen tratos diferenciados. Al no tener contratos de arriendo se genera una relación dominada por el arrendador, y esto viene acompañado de limitaciones al acceso a servicios básicos. El arriendo compartido incluso se cobra por persona, no necesariamente por pieza. Además, la falta de equipamiento o el deterioro habitacional hace que el arrendatario migrante tenga que asumir gastos mensuales para el mejoramiento de la vivienda.

"Teníamos una sola pieza y teníamos (...) tres camas, y ahí dormíamos, por decir, en una cama dormíamos puros hombres, nosotros somos tres hombres. Y después dormían dos mujeres y así, pero toda la familia amontonada." (Rosa, 28 años, boliviana).

\section{EL DESPLAZAMIENTO URBANO AL CAMPAMENTO}

Llegar a vivir en un campamento para los entrevistados responde al resultado de un conjunto de dificultades en los horizontes de su experiencia migratoria. Principalmente, los efectos del mercado del subarriendo, expresado en los altos costos, la discriminación del propietario y el deterioro habitacional. Pero también un estado de fragilidad permanente por la vulnerabilidad social que vive el migrante en el acceso al trabajo y oportunidades en la ciudad. La llegada al campamento dista mucho del imaginario urbano de las tomas de terreno 
TABLA 3. RESUMEN COMPARATIVO DE SITUACIONES RESIDENCIALES DE MIGRANTES.

\begin{tabular}{lll}
\hline Categoría & Vivienda precaria & Tránsito al campamento \\
\hline Tenencia de la vivienda & Subarriendo de vivienda & Ocupación irregular de terreno \\
\hline Uso del suelo & Zonas residenciales & Zonas no edificables \\
\hline Trato igualitario & Discriminación racial del propietario & Estigma por vivir en campamento \\
\hline Reagrupamiento familiar & Restricción a nuevos residentes & $\begin{array}{l}\text { Loteo irregular permite } \\
\text { reagrupamiento }\end{array}$ \\
\hline Servicios básicos & Formalidad limitada & Conexiones informales \\
\hline Gastos en vivienda & Altos costos en arriendo & Gastos en instalación \\
\hline Mejoramiento de la vivienda & Espacio limitado para la construcción & Posibilidad de autoconstrucción \\
\hline Redes sociales & Fragilidad relaciones sociales & Mantención de vínculos comunitarios \\
\hline Asociatividad y entorno & Deterioro de la vida barrial & $\begin{array}{l}\text { Expresión de costumbres y } \\
\text { tradiciones }\end{array}$ \\
\hline Localización & Centro & Periferia
\end{tabular}

Fuente: elaboración propia.

del siglo pasado. El proceso de "campamentación" representa actualmente una decisión que implica un desplazamiento progresivo, contiene gastos económicos, incluso la aprobación de los dirigentes de entrar al campamento. Si bien ya no habrá necesariamente un pago de arriendo, en el campamento también opera una microeconomía de uso del suelo, con gastos significativos en la instalación de la futura casa, la remoción de escombros, o para aplanar el terreno.

\begin{abstract}
"Vivir en campamentos me da facilidad para seguir mandando su ayuda para mis hijos que están allá. Menos mal que acá no pagamos por una casa. No pagamos nada. Porque si no ¿cómo yo estuviera?" (Helenis, 34 años, colombiana).

El campamento entonces adquiere una dimensión de territorio de encuentro, lugar para construir formas de habitar propios del hogar, en relaciones sociales más cercanas, que hace posible el envío de remesas sin las dificultades implícitas al subarriendo
\end{abstract}


al interior de la ciudad. Además, se pueden proyectar expectativas de vida en Chile, construir viviendas, ampliar libremente las casas, piezas para los parientes que están por llegar, se recrean los espacios domésticos, la terraza para el encuentro con los vecinos, las parrillas y mesas fuera de la vivienda.

Tal como lo señala la literatura relacionada al fenómeno,

"[l]a formación de una comunidad de migrantes propiamente tal, y en su expresión más tangible de un enclave territorial, es uno de los efectos de la marginalización social que muchos inmigrantes experimentan. La comunidad proporciona un 'refugio' de ideas, símbolos y evaluaciones compartidas, que se traduce en la expresión de una identidad colectiva" (Mora, 2009, p. 131).

Sin embargo, aparece también el estigma de vivir en campamento. El lugar donde se habita resignifica nuevos procesos de exclusión, donde el transporte de los colectivos de la ciudad no llega a los cerros donde se ubican los asentamientos, los barrios aledaños discriminan al poblador de campamento, e incluso la búsqueda de un trabajo se ve truncada por declarar el lugar donde se vive. Esto lleva en ocasiones a la negación del territorio para no alejar ni sentirse discriminado.

“iLa gente misma! Bueno, pucha, tú quieres decirle a alguien, salís al centro y quieres traerte un taxi, 'ah, ¿a las tomas? no voy pa' allá, no es que pa' allá no voy ¿me entiende?'” (Rogelio, 27 años, boliviano).
Por tanto, el campamento es una salida al estado de arriendo habitacional precario y desregulado, una salida que no necesariamente cumple con satisfacer las necesidades en cuanto a la calidad de vida y reconocimiento. La tenencia de la nueva vivienda se realiza a partir de una ocupación irregular de un terreno. Esta forma de habitar sigue vulnerando el derecho a la vivienda, pero también en la seguridad de la tenencia, con el riesgo permanente del desalojo por parte del gobierno.

"Dificultades porque, pues, no tenemos agua, dificultades porque a veces no tenemos luz, a veces que se cortó, que se arrancó una cosa u otra arreglar pero lo demás no, no. Yo ya sé que agua no tenemos yo lleno mi estanque y trato como de cohibirme de mantener esa agua para ocho días que me vuelvan a dar." (Horacio, 30 años, colombiano).

En un análisis de fuentes cartográficas de riesgo socionatural y realizando una comparación con la localización de campamentos (CIS TECHO-Chile, 2017), un 50\% de las familias en campamentos de Antofagasta habitan en zonas de remoción de masas, y la totalidad de los campamentos estudiados en el presente trabajo se encuentran en este tipo de zonas.

"A mí me da mucho miedo, porque han dicho que van a desalojar todo el campamento; pero, si voy a vivir en un lugar, que sea al menos digno, yo pienso así... que sea un lugar adecuado." (María Belén, 33 años, colombiana). 


\section{Síntesis de resultados}

Los datos muestran que el desplazamiento urbano por exclusión para la población migrante tiene una condición dinámica. Comienza por las dificultades que viven las personas en su proceso de regularización migratoria, aspecto que favorece la entrada al mercado informal del trabajo. Posteriormente, la situación laboral manifiesta repercusiones en su calidad de vida, por el abuso que enfrentan en el espacio del trabajo dado, entre otros factores, por su condición racial y los bajos salarios recibidos, que no permiten cubrir los altos costos de vida en la ciudad y especialmente de vivienda. En esta suma de factores, la vulnerabilidad social del migrante se agudiza, lo que -gatillado por algún suceso de crisis o problema, como la pérdida del trabajo, el aumento del precio del alquiler, entre otros, y sumado a la presencia de redes de contactos- provoca la llegada al campamento como una forma de desplazamiento urbano por exclusión social.

Se produce para el migrante, entonces, un transitar entre una situación de precariedad a una situación de exclusión, donde aspectos como subarriendo es reemplazado por ocupación irregular del suelo, la discriminación racial es reemplazada por el estigma territorial, y los altos costos de vivienda son reemplazados por la posibilidad de la autoconstrucción, pero en una situación de riesgo socionatural (Tabla 3). Los datos confirman la tesis de Morales et al. (2017) relativa a que, con la campamentación, el sujeto permanece funcionalmente en la ciudad, en sus márgenes físicos, pero sin pertenecer plenamente a la sociedad.

\section{Conclusiones}

Los migrantes en Chile, tras su llegada al campamento, experimentan una forma particular de hábitat residencial. Por una parte, deben enfrentar inseguridad en la tenencia, informalidad en los servicios básicos y el estigma de vivir en campamento. Sin embargo, el grado de vulnerabilidad o precariedad que poseen los migrantes en situación de campamentos es alto, y se debe, en gran medida, a que las redes que poseen en el país -ya sean familiares, sociales o institucionales- son más débiles, dificultando las posibilidades de mejorar su situación. A esto se suma el desconocimiento sobre la oferta de bienes y servicios públicos, y a las dificultades para acceder a esta oferta. Si bien los inmigrantes generan redes y desarrollan lazos fuertes dentro de los mismos asentamientos y se relacionan con organizaciones de la sociedad civil, estas se consolidan habitando en asentamientos, y debido a su tiempo de estadía en el país, no son lo suficientemente fuertes y son altamente diluidas al exterior de la unidad espacial "campamento". 
Al mismo tiempo, sin embargo, se produce una dualidad. El migrante descubre condiciones de posibilidad para autoconstruir su hábitat, expresar sus costumbres y tradiciones, mejorar el flujo del envío de remesas, la posibilidad de proyectar sus modos de habitar en Chile, en contraposición a las prácticas de violencia y abuso en la experiencia del subarriendo que impera en el centro de la ciudad. El campamento provee incluso la posibilidad de plantear la reunificación familiar en el lugar, es decir, el espacio campamento no solo implicarían nuevas formas de exclusión, sino también avances en la integración de las personas.

Es imposible desconocer un factor insuficientemente explorado en este artículo, pero que guarda relación con el grado de naturalización que tiene la figura del asentamiento informal para sujetos provenientes de entornos urbanos donde la modalidad de ocupación informal del suelo urbano es la norma para los más pobres; esto es especialmente gravitante en países de la órbita andina y del Caribe. Esta veta de importación cultural de una cierta identidad del campamento, o transculturización del concepto de "campamento" al fin y al cabo, es un fenómeno que debe ser mejor estudiado y comprendido. La connotación misma de las palabras "campamento" o "informalidad" parecieran tensionarse al referirnos a hábitats que para muchos podrían encontrarse perfectamente normalizados, y para quienes, desde su cotidianeidad, se trataría simplemente de "barrio".
El nuevo contexto de Chile muestra la existencia de una alta migración, sumado a un mercado de arriendo de vivienda desregulado que funciona como un verdadero mecanismo de expulsión (López Morales, 2016). Todo ello exige consideraciones teóricas nuevas. En este sentido, el presente trabajo intentó cuestionar la vigencia de tesis explicativas de los campamentos en Chile propias de una década atrás, como por ejemplo la tesis de la campamentación como mecanismo de presión política (Brain, Prieto y Sabatini, 2010), que queda desajustada a la luz de los nuevos factores de crecida migración, etnia y raza que experimenta Chile actualmente, y que tan solo hace una década atrás eran desconocidos e imprevisibles. El trabajo complementa también la tesis del retorno al campamento visto como una resistencia a la subjetivación individualista generada por la política de vivienda social neoliberal chilena (Morales et al., 2017), con un enfoque enfocado en factores causales materiales quizá más explicativos, tales como discriminación, abuso y costos de arriendo en la ciudad formal.

El resurgimiento de campamentos en el país muestra una dualidad entre exclusión y posibilidades de integración, realidad que no solo opera para quienes optan por la vía de la "campamentación" en el acceso a la vivienda, sino que también para parte importante de la población del país. La comuna o barrio donde se vive, el salario que se gana y el colegio donde se estudia son también factores no 
solo de segmentación social, sino también a menudo de exclusión. La abismal brecha social que aún se experimenta en Chile, la falta de acceso a los recursos, empleos de calidad e ingresos suficientes (con alto nivel de deuda) para más de la mitad de chilenos, y los precarizados sistemas de apoyo estatal que nos caracterizan como país, muestran que el afloramiento de los campamentos es solo la punta de un iceberg que abarca a todo un sistema de distribución desigual de recursos, en particular el recurso de la vivienda, en uno de los países de mayor polarización social a nivel regional.

\section{Referencias bibliográficas}

Apaolaza, R. (2018). Territorio, transportes y capitales. Dinámicas y efectos del aislamiento socioterritorial sobre los jóvenes residentes de los nuevos asentamientos periféricos del área metropolitana de Buenos Aires (Tesis Doctoral, sin publicar). Universidad de Buenos Aires, Buenos Aires.

Banco Central de Chile (2015). Estadísticas producto interno bruto. Chile. Santiago: Autor.

Besoain, C. y Cornejo, M. (2015) Vivienda social y subjetivación urbana en Santiago de Chile: espacio privado, repliegue presentista y añoranza. Psicoperspectivas, 14(2), 16-27. https://doi.org/10.5027/ PSICOPERSPECTIVAS-VOL14-ISSUE2-FULLTEXT-369.
Brain, I., Prieto, J., y Sabatini, F. (2010). Vivir en campamentos: ¿camino hacia la vivienda formal o estrategia de localización para enfrentar la vulnerabilidad? EURE, 36(109), p. 111-141. https://doi. org/10.4067/S0250-71612010000300005.

Busso, G. (2001). Vulnerabilidad social: nociones e implicancias de políticas para Latinoamérica a inicios del siglo XXI. Presentado a Seminario Internacional "Las diferentes expresiones de la vulnerabilidad social en América Latina y el Caribe", Santiago de Chile.

Cámara Chilena de la Construcción. (2017) Balance de vivienda social y entorno urbano 2017. Santiago: CCHC. Recuperado de http://www.cchc. cl/uploads/archivos/archivos/BalanceVivienda_2017.pdf.

Castells, M. (1974). Estructura de clases y politica urbana en América Latina. [Buenos Aires]: Ediciones SIAP.

CIS TECHO-Chile. (2015). Datos duros de una realidad mucho más dura: Encuesta Nacional de Campamentos 2015. Santiago: Autor.

CIS TECHO-Chile. (2016). Campamentos sin fronteras: estudio cualitativo sobre población extranjera en campamentos de Antofagasta. Santiago: Autor.

CIS TECHO-Chile. (2017). Actualización catastro nacional de campamentos. Santiago: Autor.

Daher, A. (2003). Regiones-commodities: crisis y contagio en Chile. EURE, 29(86), 89-108. https://doi. org/10.4067/S0250-71612003008600005. 
Farha, L. (2017). Declaración final de la misión de la Relatora Especial sobre la vivienda adecuada, Santiago de Chile, 28 de abril de 2017. Recuperado de http:// www.ohchr.org/SP/NewsEvents/Pages/DisplayNews.aspx? NewsID=21544\&LangID=S.

González, L. (2009). Orientaciones de lectura sobre vulnerabilidad social. En L.M. González (Comp.), Lecturas sobre vulnerabilidad y desigualdad social (pp. 13-29). Córdoba: Universidad Nacional de Córdoba.

GORE Antofagasta. (2015). Estadísticas de población. Chile: Gobierno Regional de Antofagasta.

Hernández, R., Fernández, C., y Baptista, P. (2010). Metodologías de la investigación (5a ed.). México: Mc Graw Hill.

Instituto Nacional de Estadísticas. (2017). Resultados censo de población y vivienda 2017. Santiago: Autor.

Kaztman, R. (1999). Activos y estructuras de oportunidades: estudios sobre las raíces de la vulnerabilidad social en Uruguay. Montevideo: PNUD, CEPAL.

López Morales, E. (2015) Assessing exclusionary displacement through rent gap analysis in the urban redevelopment of inner Santiago, Chile. Housing Studies, 31(5), 540-559. https://doi.org/10.1080/0 2673037.2015 .1100281 .

López Morales, E. (2016). A multidimensional approach to urban entrepreneurialism, financialization, and gentrification in the high-rise residential market of inner Santiago, Chile. En P. Zarembka (Series Ed.), Research in Political Economy: Vol. 31 Risking
Capitalism, (pp. 79-105). https://doi.org/10.1108/ S0161-723020160000031005.

Méndez, M., Otero, G., López, E., Link, F., y Castillo, V. (2017). Ciudad, conflicto y cohesión social: sobre la desigualdad socio-espacial en el Chile actual. Santiago, Chile: COES. Recuperado de https://www. coes.cl/wp-content/uploads/2017/08/N10-ELSOC-MOD3-TERRITORIO.pdf.

Ministerio de Desarrollo Social. (2015). Resultados encuesta CASEN. Chile: Autor.

Ministerio de Vivienda y Urbanismo. (2011). Catastro nacional de campamentos. Chile: Autor.

Mora, C. (2009). Estratificación social y migración intrarregional: algunas caracterizaciones de la experiencia migratoria en Latinoamérica. Universum, 24(1), 128-143. https://doi.org/10.4067/ S0718-23762009000100008.

Morales, R., Besoain, C., Soto, A., Pinto, L., Hidalgo, K., Fernández, I., y Bernal, V. (2017). Retorno al campamento: resistencia y melancolía en los márgenes de la ciudad formal. Revista INVI, 32(90), 51-75. https://doi.org/10.4067/ S0718-83582017000200051.

Moreno Crossley, J. (2008). El concepto de vulnerabilidad social en el debate en torno a la desigualdad: problemas, alcances y perspectivas. Center for Latin American Studies University of Miami.

Rivas, A. (2013). Campamentos: factores socioespaciales vinculados a su persistencia. (Tesis de Magister en Urbanismo, sin publicar). Universidad de Chile, 
Santiago. Recuperado de http://repositorio.uchile. cl/handle/2250/116946.

Rodríguez, A. y Sugranyes, A. (2005). Los con techo. Un desafío para la política de vivienda social. Santiago: Ediciones SUR.

Sabatini, F. (2017, 28 de julio). Guetos verticales: ¿densidad o discriminación? El Mostrador. Recuperado de http://www.elmostrador.cl/noticias/opinion/2017/07/28/ guetos-verticales-densidad-o-discriminacion/.

Slater, T. (2009). Missing Marcuse: On gentrification and displacement. City, 13(2-3), 292-311. https:// doi.org/10.1080/13604810902982250.

Solimano, A. (2008). Migraciones internacionales en América Latina: booms, crisis y desarrollo. Santiago: Fondo de Cultura Económica.

Stefoni, C., Leiva, S., y Bonhomme, M. (2017). Migración internacional y precariedad laboral. El caso de la industria de la construcción en Chile. REMHU-Revista Interdisciplinar da Mobilidade Humana, 25(49), 95-112. https://doi. org/10.1590/1980-85852503880004906.

Subirats, J. (2004). Pobreza y exclusión social. Un análisis de la realidad española y europea. Barcelona: Fundación La Caixa.

Thodes Miranda, E. (2016). Segregación socioespacial en ciudades mineras: el caso de Antofagasta, Chile. Notas de población, (102), 203-227.

Toro, F. y Orozco, H. (2018). Concentración y homogeneidad socioeconómica: representación de la segregación urbana en seis ciudades intermedias de Chile. Revista de Urbanismo, (38), 1-21. https:// doi.org/10.5354/0717-5051.2018.48834.

Vekemans, R. y Fuenzalida, I. (1976). Marginalidad, promoción popular y neo-marxismo: críticas y contracríticas. Bogotá: Cedial. 